\title{
A consistency result on weak reflection
}

\author{
by
James Cummings (Jerusalem), Mirna Džamonja (Jerusalem) and Saharon Shelah (Jerusalem and Brunswick, N.J.)

\begin{abstract}
In this paper we study the notion of strong non-reflection, and its contrapositive weak reflection. We say $\theta$ strongly non-reflects at $\lambda$ iff there is a function $F: \theta \rightarrow \lambda$ such that for all $\alpha<\theta$ with $\operatorname{cf}(\alpha)=\lambda$ there is $C$ club in $\alpha$ such that $F\lceil C$ is strictly increasing. We prove that it is consistent to have a cardinal $\theta$ such that strong non-reflection and weak reflection each hold on an unbounded set of cardinals less than $\theta$.
\end{abstract}

1. Introduction. In this paper we study the notion of strong nonreflection, which was introduced in [4] and is further studied in [3]. We prove that for a fixed $\theta$ we can have an unbounded set of cofinalities at which strong non-reflection holds, and an unbounded set where it fails.

Definition 1. Let $\theta$ be a regular cardinal, and let $\lambda$ be an ordinal with $\lambda \geq \theta$

- $S_{\theta}^{\lambda}=\{\alpha<\lambda \mid \operatorname{cf}(\alpha)=\theta\}$.

- $S_{<\theta}^{\lambda}=\{\alpha<\lambda \mid \operatorname{cf}(\alpha)<\theta\}$.

- If $\theta$ is uncountable, then $\lambda$ strongly non-reflects at $\theta$ iff there is a function $F: \lambda \rightarrow \theta$ such that for all $\alpha \in S_{\theta}^{\lambda}$ there is $C$ club in $\alpha$ such that $F\lceil C$ is strictly increasing. We will write $\operatorname{SNR}(\lambda, \theta)$ for this.

- $\lambda$ weakly reflects at $\theta$ iff $\lambda$ does not strongly non-reflect at $\theta$.

In [4] Džamonja and Shelah prove some theorems connecting weak club principles, saturated ideals, and the ideal $\mathcal{I}[\lambda, \theta)$ consisting of those $A \subseteq \lambda$ such that there is $h: \lambda \rightarrow \theta$ increasing on a club at every point of $A \cap S_{\theta}^{\lambda}$.

1991 Mathematics Subject Classification: 03E35, 03E55, 03E99.

The first author was supported by a Postdoctoral Fellowship at the Hebrew University.

The second author was partially supported by the Basic Research Fund of the Israel Academy of Science and a Postdoctoral Fellowship from the Hebrew University.

The third author was partially supported by the Basic Research Fund of the Israel Academy of Science. Paper number 571.

The research for this paper was done in the period July 1994 - January 1995. 
In particular, Theorem 2.5 of that paper shows that a certain weak club principle is incompatible with saturated ideals at successors of singulars, and Theorem 2.8 connects weak reflection and the weak club principle.

In [3] strong non-reflection is used as a tool to show that different instances of stationary reflection are independent. For example, it is shown there that "every stationary subset of $S_{\aleph_{0}}^{\aleph_{3}}$ reflects at a point in $S_{\aleph_{2}}^{\aleph_{3}}$ " is consistent with "every stationary subset of $S_{\aleph_{1}}^{\aleph_{3}}$ has a non-reflecting stationary subset".

We make a few remarks about the definitions. The next lemma is implicit in Observation 1.2.3 of [4].

Lemma 1. Let $\lambda$ be an ordinal, and $\left\langle\lambda_{i}: i<\lambda\right\rangle$ a $\lambda$-sequence of ordinals with $\operatorname{cf}\left(\lambda_{i}\right) \neq \theta$. Let $F: \lambda \rightarrow \theta$ and $F_{i}: \lambda_{i} \rightarrow \theta$ witness strong non-reflection at $\theta$ for $\lambda$ and each of the $\lambda_{i}$. Then there is $G: \sum_{i<\lambda} \lambda_{i} \rightarrow \theta$ witnessing strong non-reflection for $\sum_{i<\lambda} \lambda_{i}$.

Proof. Define $G\left(\sum_{i<j} \lambda_{i}\right)=F(j)$ and $G\left(\sum_{i<j} \lambda_{i}+\nu\right)=F_{j}(\nu)$ for $0<\nu<\lambda_{j}$. It is easy to check that this works.

It is proved in [4], using the previous lemma, that the least $\lambda$ which weakly reflects at $\theta$ is a regular cardinal greater than $\theta$.

As the terminology suggests, there is a connection between weak reflection and the more familiar notion of stationary reflection.

Definition 2. Let $\kappa<\mu<\nu$ be regular cardinals. Then $\operatorname{Ref}(\nu, \mu, \kappa)$ iff for every stationary $S \subseteq S_{\kappa}^{\nu}$ there is $\alpha \in S_{\mu}^{\nu}$ such that $S \cap \alpha$ is stationary in $\alpha$. We will also use $\operatorname{Ref}(\nu, \mu,<\kappa)$ as a shorthand for " $\forall \delta \in \operatorname{REG} \cap \kappa$ $\operatorname{Ref}(\nu, \mu, \delta) "$.

The next fact shows that strong non-reflection at $\theta$ is antithetical to stationary reflection to points of cofinality $\theta$.

LEMma 2. Suppose that $\lambda$ strongly non-reflects at $\theta$. Then for every stationary $S \subseteq \lambda$ there is $T \subseteq S$ stationary such that $T \cap \alpha$ is non-stationary for all $\alpha \in S_{\theta}^{\lambda}$.

Pr o of. Use Fodor's Lemma to find $T$ on which $F: \lambda \rightarrow \theta$ witnessing the strong non-reflection is constant. If $C \subseteq \alpha$ is a club on which $F$ is strictly increasing then $C$ meets $T$ in at most one point.

It is not hard to see that if $\left\langle C_{\alpha}: \alpha<\kappa^{+}\right\rangle$is a $\square_{\kappa}$-sequence then the function $F: \alpha \mapsto$ o.t. $\left(C_{\alpha}\right)$ witnesses that $\kappa^{+}$strongly non-reflects at $\kappa$. More is true, see 1.7 of [4]. The following remark is immediate from the definition.

Lemma 3. If $\theta=\operatorname{cf}(\theta)<\lambda<\lambda^{*}$, and $\lambda$ weakly reflects at $\theta$, then $\lambda^{*}$ weakly reflects at $\theta$.

We are now ready to state the main result. 
TheOREM 1. Let GCH hold, let $\theta$ be regular, and suppose that there are cardinals $\left\langle\lambda_{i}, \theta_{i}, \kappa_{i}: i<\theta\right\rangle$ such that for all $i<\theta$,

(1) $\lambda_{i}=\operatorname{cf}\left(\lambda_{i}\right)<\lambda_{i}^{+}<\theta_{i}=\operatorname{cf}\left(\theta_{i}\right)<\kappa_{i}$, and $\kappa_{i}$ is measurable.

(2) $\lambda_{i}>\left(\sup \left\{\kappa_{j} \mid j<i\right\}\right)^{++}$.

Then there is a generic extension in which $\operatorname{SNR}\left(\theta, \lambda_{i}\right)$ and $\operatorname{Ref}\left(\kappa_{i}, \theta_{i}\right.$, $\left.<\theta_{i}\right)$ hold for every $i$. In particular, by Lemmas 2 and $3, \theta$ weakly reflects at $\theta_{i}$.

The proof will involve two stages. First we force functions that witness the strong non-reflection at the points $\lambda_{i}$, via an iterated forcing with Easton support. An important feature of the construction is that the individual steps in the forcing have an increasing degree of strategic closure, and at any stage a witness to the desired degree of strategic closure was added by the previous stages.

We will show that the first stage preserves the measurability of all the $\kappa_{i}$. In the second stage we will force with a product of the Lévy collapses $\operatorname{Coll}\left(\theta_{i},<\kappa_{i}\right)$, and use Baumgartner's argument from [2] to show that $\operatorname{Ref}\left(\kappa_{i}, \theta_{i},<\theta_{i}\right)$ holds in the extension.

2. Forcing strong non-reflection. Let $\sigma$ and $\lambda$ be regular cardinals with $\sigma<\lambda$. In this section we define a forcing $\mathbb{P}(\sigma, \lambda)$ which adds a function from $\lambda$ to $\sigma$ witnessing strong non-reflection for $\lambda$ at $\sigma$. We could make the same definition for $\lambda$ an arbitrary ordinal greater than $\sigma$, but for our purposes it will suffice to restrict ourselves to regular cardinals.

Definition 3. Conditions in $\mathbb{P}(\sigma, \lambda)$ are functions $p$ such that $\operatorname{dom}(p)$ $<\lambda, \operatorname{rge}(p) \subseteq \sigma$, and for every $\beta \leq \operatorname{dom}(p)$ if $\beta \in S_{\sigma}^{\lambda}$ then there is a club $C$ in $\beta$ such that $p\lceil C$ is strictly increasing.

The condition $p$ extends the condition $q$ iff $p \supseteq q$. We write this as $p \leq q$.

Clearly this forcing has at most $\sigma^{<\lambda}$ conditions, so enjoys the $\left(\sigma^{<\lambda}\right)^{+}$-c.c. There are several pieces of information about the closure properties of the forcing that we will need later.

LEMMA 4. Let $\left\langle p_{\alpha}: \alpha<\varrho\right\rangle$ be a strictly decreasing sequence of conditions in $\mathbb{P}(\sigma, \lambda)$, where $\varrho \in S_{\neq \sigma}^{\lambda}$. Then $p=\bigcup_{\alpha} p_{\alpha}$ is the greatest lower bound for the sequence.

Proof. Notice that $\operatorname{cf}(\operatorname{dom}(p))=\operatorname{cf}(\varrho) \neq \sigma$, so that if $\beta \leq \operatorname{dom}(p)$ and $\operatorname{cf}(\beta)=\sigma$ then $\beta \in \operatorname{dom}\left(p_{\alpha}\right)$ for some $\alpha<\varrho$. There is $C \operatorname{club}$ in $\beta$ such that $p\left\lceil C=p_{\alpha}\lceil C\right.$ is strictly increasing.

We remind the reader of the notion of strategic closure. 
Definition 4. Let $\mathbb{P}$ be a partial ordering, and let $\eta$ be an ordinal.

(1) The game $G(\mathbb{P}, \eta)$ is played by two players I and II, who take turns to play elements $p_{\alpha}$ of $\mathbb{P}$ for $0<\alpha<\eta$, with player I playing at odd stages and player II at even stages (nota bene: limit ordinals are even).

The rules of the game are that the sequence that is played must be decreasing (not necessarily strictly decreasing), the first player who cannot make a move loses, and player II wins if play proceeds for $\eta$ stages.

(2) $\mathbb{P}$ is $\eta$-strategically closed iff player II has a winning strategy in $G(\mathbb{P}, \eta)$.

(3) $\mathbb{P}$ is $<\eta$-strategically closed iff for all $\zeta<\eta$, $\mathbb{P}$ is $\zeta$-strategically closed.

We say that a forcing notion $\mathbb{P}$ is $<\lambda$-distributive iff it does not add any $<\lambda$-sequence of ordinals to the ground model (equivalently, the intersection of fewer than $\lambda$ dense open sets is non-empty). The following lemma is easy.

Lemma 5. If $\mathbb{P}$ is $<\lambda$-closed it is $\lambda$-strategically closed, and if $\mathbb{P}$ is $<\lambda$-strategically closed it is $<\lambda$-distributive.

Notice that $\mathbb{P}(\sigma, \lambda)$ will only contain conditions of lengths unbounded in $\lambda$ if $\operatorname{SNR}(\mu, \sigma)$ holds for all $\mu<\lambda$. This condition is actually enough to make $\mathbb{P}(\sigma, \lambda)<\lambda$-strategically closed.

Lemma 6. Suppose that all $\mu \in[\sigma, \lambda)$ are strongly non-reflecting at $\sigma$. Then $\mathbb{P}(\sigma, \lambda)$ is $<\lambda$-strategically closed.

Proof. Let $\eta<\lambda$. If $\eta<\sigma$ then player II can win with the following strategy: he plays $p_{2 \gamma}:=\bigcup_{\alpha<2 \gamma} p_{\alpha}$.

If $\sigma \leq \eta<\lambda$ then by hypothesis there is a function $F: \eta \rightarrow \sigma$ witnessing strong non-reflection. Player II will play $p_{2 \gamma}:=\left(\bigcup_{\alpha<2 \gamma} p_{\alpha}\right) \frown F(\gamma)$. We check that this is a winning strategy.

Let $2 \delta$ be an even stage of cofinality $\sigma$ in $G(\mathbb{P}(\sigma, \lambda), \eta)$. There is $D$ club in $\delta$ such that $F\left\lceil D\right.$ is strictly increasing. If we define $C=\left\{\ln \left(p_{2 \gamma}\right) \mid \gamma \in D\right\}$ then $C$ witnesses that II does not lose at stage $2 \delta$.

We will be interested in forcing strong non-reflection to several values of $\sigma$ simultaneously. For this we will use a certain dense subset of the $<\lambda$-support product of the appropriate $\mathbb{P}(\sigma, \lambda)$.

Definition 5. Let $A \subseteq \operatorname{REG} \cap \lambda$. Then $\mathbb{P}(A, \lambda)$ is the set of functions $p$ such that

(1) $\operatorname{dom}(p)=(A \cap \gamma) \times \gamma$ for some $\gamma<\lambda$.

(2) If $\operatorname{dom}(p)=(A \cap \gamma) \times \gamma$ and $\sigma \in A \cap \gamma$ then $\alpha<\gamma \mapsto p(\sigma, \alpha)$ is a condition in $\mathbb{P}(\sigma, \lambda)$.

If $p, q \in \mathbb{P}(A, \lambda)$ then $p \leq q$ iff $p$ extends $q$. 
Clearly $|\mathbb{P}(A, \lambda)| \leq \lambda^{<\lambda}$, so the forcing has the $\left(\lambda^{<\lambda}\right)^{+}$-c.c. We also record some information about the closure of the forcing.

Lemma 7. Let $\left\langle p_{\alpha}: \alpha<\varrho\right\rangle$ be a strictly decreasing sequence of conditions in $\mathbb{P}(A, \lambda)$, where $\operatorname{cf}(\varrho) \notin A$. Then the condition $p$ given by $p(i):=$ $\bigcup_{\alpha<\varrho} p_{\alpha}(i)$ is the greatest lower bound for the sequence.

The next lemma is easy, with a proof almost identical to that of Lemma 6.

Lemma 8. Let $A$ and $\lambda$ be as above. Suppose that for all $\sigma \in A$, all $\mu \in[\sigma, \lambda)$ are strongly non-reflecting at $\sigma$. Then $\mathbb{P}(A, \lambda)$ is $<\lambda$-strategically closed.

3. The iteration. The idea of the construction is now to define $A=$ $\left\{\lambda_{i} \mid i<\theta\right\}$ (where the $\lambda_{i}$ are as in the statement of Theorem 1) and to iterate $\mathbb{P}(A \cap \lambda, \lambda)$ for all regular $\lambda \leq \theta$. A crucial point will be that the forcing at stage $\lambda$ is $<\lambda$-strategically closed, using Lemma 8 and the fact that in the iteration we have already arranged the required instances of non-reflection below $\lambda$.

We will do a "Reverse Easton" iteration, that is to say, an iteration where direct limits are taken at strongly inaccessible limit stages and inverse limits are taken at other limit stages. We will refer to [1] for details about this sort of iteration, and we will also follow the notation of that paper (in particular, $\mathbb{P}_{\alpha}$ is the forcing up to stage $\alpha$ and $\dot{\mathbb{Q}}_{\alpha} \in V^{\mathbb{P}_{\alpha}}$ is the forcing at $\alpha$ ).

Formally, we will define $\dot{\mathbb{Q}}_{\alpha}$ to be $\{0\}$ if $\alpha$ is not a regular cardinal, and to be $\mathbb{P}(A \cap \alpha, \alpha)_{V^{\mathbb{P}} \alpha}$ if $\alpha$ is regular. We will collect some information about the iteration in the following lemma.

Lemma 9. Let $\mathbb{P}_{\alpha}$ and $\dot{\mathbb{Q}}_{\alpha}$ be as above, and let $\dot{\mathbb{R}}_{\beta, \alpha}$ be the canonical iteration in $V^{\mathbb{P}_{\beta}}$ such that $\mathbb{P}_{\beta} * \dot{\mathbb{R}}_{\beta, \alpha}$ has a dense subset isomorphic to $\mathbb{P}_{\alpha}$. Then for all regular $\alpha \leq \theta$,

(1) $\left|\mathbb{P}_{\alpha}\right| \leq \alpha$.

(2) $V^{\mathbb{P}_{\alpha}} \models G C H$, so in particular $V^{\mathbb{P}_{\alpha}} \models\left|\dot{\mathbb{Q}}_{\alpha}\right|=\alpha$.

(3) $\mathbb{P}_{\alpha+1}$ has the $\alpha^{+}$-c.c. In addition, if $\alpha$ is Mahlo, then $\mathbb{P}_{\alpha}$ has the $\alpha-c . c$.

(4) $V^{\mathbb{P}_{\alpha}} \models \dot{\mathbb{Q}}_{\alpha}$ is $<\alpha$-strategically closed.

(5) For all regular $\beta<\alpha, \mathbb{R}_{\beta, \alpha}$ is $<\beta$-strategically closed in $V^{\mathbb{P}_{\beta}}$.

(6) $\mathbb{P}_{\alpha}$ preserves all cardinals and cofinalities.

Pr o of. The proof will be by induction on $\alpha$. Most of the proof is straightforward, using the results of Section 2 in [1] to power the induction. The distinctive point here is in showing that clauses (4) and (5) hold at $\alpha$, given that we have proved the lemma for regular cardinals less than $\alpha$.

By construction, $\mathbb{P}_{\alpha}$ forces that for every $\lambda \in A$ and every regular cardinal $\beta \in[\lambda, \alpha)$ we have $\operatorname{SNR}(\beta, \lambda)$. As we remarked after Lemma 1 , this 
implies that for every ordinal $\gamma \in[\lambda, \alpha)$ we have $\operatorname{SNR}(\gamma, \lambda)$. By Lemma 8 this means that $\mathbb{P}(A \cap \alpha, \alpha)$ is $<\alpha$-strategically closed in $V^{\mathbb{P}_{\alpha}}$.

Finally, to see that clause (5) holds one should check that Theorem 2.5 of [1] is still true if " $\kappa$-closed" is replaced by " $<\kappa$-strategically closed". This is routine, the point is that a term for a strategy can be applied to a term for a condition to get a term for a stronger condition.

We make some remarks about this construction.

(1) Since cardinals and cofinalities are preserved, a witness to strong non-reflection added at some stage by some $\mathbb{P}(\sigma, \lambda)$ will remain a witness at all subsequent stages.

(2) At stage $\theta$ we forced with $\mathbb{P}(A, \theta)$, so added witnesses to all the strong non-reflection that is claimed in Theorem 1.

4. Preserving measurability. As we mentioned in the first section, we want to show that for each $i$ the measurability of $\kappa_{i}$ is preserved by the iteration $\mathbb{P}_{\theta}$. It is enough to argue that $\kappa_{i}$ is measurable in the extension by $\mathbb{P}_{\kappa_{i}^{+}+1}$, because the rest of the forcing is $\kappa_{i}^{+}$-strategically closed, so that the power set of $\kappa_{i}$ does not change and a measure remains a measure. For brevity, we will denote $\kappa_{i}$ by $\kappa$ throughout this section.

Let $G$ be $\mathbb{P}_{\kappa}$-generic over $V$, let $g$ be $\mathbb{P}(A \cap \kappa, \kappa)$-generic over $V[G]$, and let $h$ be $\mathbb{P}\left(A \cap \kappa^{+}, \kappa^{+}\right)$-generic over $V[G][g]$. Let $j: V \rightarrow M$ be the ultrapower map arising from a normal measure $U$ on $\kappa$. We list some facts about $j$ and $M$, all of whose proofs can be found in [5].

(1) $\operatorname{crit}(j)=\kappa$.

(2) ${ }^{\kappa} M \subseteq M$.

(3) $H_{\kappa^{+}} \subseteq M$.

(4) $\kappa^{+}=\kappa_{M}^{+}$.

(5) $\kappa^{+}<j(\kappa)<j\left(\kappa^{+}\right)<\kappa^{++}$.

(6) $M=\{j(F)(\kappa) \mid F \in V$ and $\operatorname{dom}(F)=\kappa\}$.

The strategy of the proof will be to define, in $V[G][g][h]$, an extension of $j: V \rightarrow M$ to a new embedding $j: V[G][g][h] \rightarrow N \subseteq V[G][g][h]$. The existence of such an extension will imply that $\kappa$ is still measurable in $V[G][g][h]$.

We start by comparing the iterations $\mathbb{P}_{\kappa^{+}+1}$ and $j\left(\mathbb{P}_{\kappa^{+}+1}\right)$. The forcing $j\left(\mathbb{P}_{\kappa^{+}+1}\right)$ is an iteration defined in $M$, forcing strong non-reflection at cofinalities in the set $j(A) \cap j\left(\kappa^{+}\right)$for all $M$-regular cardinals up to $j\left(\kappa^{+}\right)$. Since $A \cap \kappa$ is bounded in $\kappa$ and $\lambda_{i+1}>\kappa^{+}$, we see that

$$
A \cap\left(\kappa^{+}+1\right)=j(A) \cap\left(j\left(\kappa^{+}\right)+1\right)=A \cap \kappa .
$$

By the resemblance between $V$ and $M$, if we compute the iteration $j\left(\mathbb{P}_{\kappa^{+}+1}\right)$ up to stage $\kappa^{+}$we get $\mathbb{P}_{\kappa^{+}+1}$. We can therefore compute a generic 
extension $M[G][g][h]$ of $M$ by using the $V$-generic filters, and observing that $V$-generic filters are $M$-generic.

We claim that $V[G][g][h] \models{ }^{\kappa}(M[G][g][h]) \subseteq M[G][g][h]$. Since $\mathbb{P}_{\kappa+1}$ is $\kappa^{+}$-c.c. every canonical $\mathbb{P}_{\kappa+1}$-name for a $\kappa$-sequence of ordinals is in $M$, so that easily $V[G][g] \models{ }^{\kappa}(M[G][g]) \subseteq M[G][g]$. The forcing $\mathbb{Q}_{\kappa^{+}}$is $<\kappa^{+}$strategically closed in $V[G][g]$, so it adds no $\kappa$-sequence of ordinals, and we are done.

In $M[G][g][h]$ let $\mathbb{R}=\mathbb{R}_{\kappa^{+}+1, j(\kappa)}$ be the canonical factor forcing to prolong $G * g * h$ to a $j\left(\mathbb{P}_{\kappa}\right)$-generic. We claim that $\mathbb{R}$ is $\kappa^{+}$-strategically closed in $V[G][g][h]$. This follows from the fact that $\mathbb{R}$ is $<\kappa_{M}^{++}$-strategically closed in $M[G][g][h]$ and the fact that $V[G][g][h] \models{ }^{\kappa}(M[G][g][h]) \subseteq M[G][g][h]$. The point is that if player II plays for $\kappa^{+}$steps in $V[G][g][h]$ using the strategy from $M[G][g][h]$, then every initial segment of the play is in $M[G][g][h]$, so that player II does not get stuck at any stage below $\kappa^{+}$.

The previous claim explains why we are working in $V[G][g][h]$ rather than $V[G][g]$. If we truncate $j\left(\mathbb{P}_{\kappa}\right)$ at $\kappa+1$ then the rest of the forcing will be $<\kappa^{+}$-strategically closed in $V[G][g]$, but the following stage of the proof will demand $\kappa^{+}$-strategic closure.

Recall that $\kappa^{+}<j(\kappa)<\kappa^{++}$. In $M[G][g][h]$ the forcing $\mathbb{R}$ is $j(\kappa)$-c.c. and has size $j(\kappa)$, so there are at most $j(\kappa)$ maximal antichains in that model. In $V[G][g][h]$ let us enumerate these antichains as $\left\langle A_{\alpha}: \alpha<\kappa^{+}\right\rangle$. Now consider a run of the game $G\left(\mathbb{R}, \kappa^{+}\right)$in which player I plays the following strategy: in response to $p_{2 \gamma}$ player I will choose some element $q_{\gamma}$ of $A_{\gamma}$ such that $p_{2 \gamma}$ is compatible with $q_{\gamma}$, and then will play $p_{2 \gamma+1}$ which is some common refinement. Player II will play according to some winning strategy; after $\kappa^{+}$steps we have built a decreasing sequence of conditions which clearly generates an $M[G][g][h]$-generic filter $H$.

Now we will start to extend $j$. Define $G^{+}:=G * g * h * H$, which will be $j\left(\mathbb{P}_{\kappa}\right)$-generic over $M$. We attempt to define $j: V[G] \rightarrow M\left[G^{+}\right]$by $j\left(\dot{\tau}^{G}\right):=j(\dot{\tau})^{G^{+}}$. We check that this is a well-defined elementary embedding, using the following well-known fact.

Lemma 10. Let $k: M \rightarrow N$ be an elementary embedding between two transitive models of $Z F C$. Let $\mathbb{P} \in M$ be some forcing, let $k(\mathbb{P})=\mathbb{Q}$, and suppose that we have $G$ which is $\mathbb{P}$-generic over $M$ and $H$ which is $\mathbb{Q}$-generic

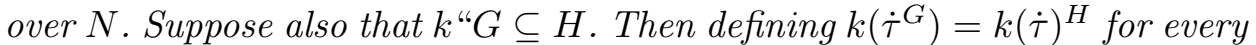
$\dot{\tau} \in M^{\mathbb{P}}$ gives a well-defined elementary embedding $k: M[G] \rightarrow N[H]$, which extends $k: M \rightarrow N$ and has $k(G)=H$.

Pr o of. Easy, using the Truth Lemma and the elementarity of $k$.

By Lemma 10, it is enough to check that $j^{\text {" }} G \subseteq G^{+}$. $G$ is generic for $\mathbb{P}_{\kappa}$, which was constructed as a direct limit, so every condition $p$ in $G$ 
has support bounded in $\kappa$. Since $\operatorname{crit}(j)=\kappa$, the condition $j(p)$ contains the same information as $p$, and since $G=G^{+} \uparrow \kappa$, we conclude that $j(p) \in G^{+}$.

Since $G^{+} \in V[G][g][h]$, we see that $M\left[G^{+}\right] \subseteq V[G][g][h]$. Also, we know that $H$ is generic for a forcing which adds no $\kappa$-sequences of ordinals over $M[G][g][h]$, so that $V[G][g][h]={ }^{\kappa}\left(M\left[G^{+}\right]\right) \subseteq M\left[G^{+}\right]$.

Now we aim to lift $j$ further to get a map with domain $V[G][g]$. In $V[G]$ the forcing $\mathbb{Q}_{\kappa}$ has cardinality $\kappa$, and is $<\kappa$-strategically closed with at most $2^{\kappa}$ (that is, $\kappa^{+}$) many maximal antichains. Since $j: V[G] \rightarrow M\left[G^{+}\right]$is elementary, in $M\left[G^{+}\right]$the forcing $\mathbb{Q}_{j(\kappa)}$ is $<j(\kappa)$-strategically closed with at most $j\left(\kappa^{+}\right)$maximal antichains.

Arguing as before, $\mathbb{Q}_{j(\kappa)}$ is $\kappa^{+}$-strategically closed in $V[G][g][h]$. Since $j\left(\kappa^{+}\right)<\kappa^{++}$we can repeat the argument from the construction of $H$ to build $g^{+}$which is $\mathbb{Q}_{j(\kappa)}$-generic over $M\left[G^{+}\right]$. But it is not clear at this point that we can lift $j$ onto $V[G][g]$, because it may not be the case that $j^{\prime} " g \subseteq g^{+}$.

We will use Silver's "master condition" idea. Observe that $g \in M\left[G^{+}\right]$, and that $g$ is equivalent to a function $p$ where $\operatorname{dom}(p)=(A \cap \kappa) \times \kappa$ and $p(\sigma,-): \alpha<\kappa \mapsto p(\sigma, \alpha)$ witnesses $\operatorname{SNR}(\kappa, \sigma)$ for each $\sigma \in A \cap \kappa$.

Recall that $\mathbb{Q}_{j(\kappa)}$ is defined to be $\mathbb{P}(j(A) \cap j(\kappa), j(\kappa))$. We claim that $p \in \mathbb{Q}_{j(\kappa)}$. The support condition is satisfied because $\kappa<j(\kappa)$ and (as we saw before) $j(A) \cap j(\kappa)=j(A \cap \kappa)=A \cap \kappa$. It is enough to show that for each $\sigma$ we have $p(\sigma,-) \in \mathbb{P}(\sigma, j(\kappa))$, which is to say that for all $\delta \leq \kappa$ of cofinality $\sigma$ there is a club in $\delta$ on which $p(\sigma,-)$ is increasing. This is easy because $V, M$ and $M\left[G^{+}\right]$agree about cardinals and cofinalities up to $\kappa^{+}$.

Since $p$ is a condition in $\mathbb{Q}_{j(\kappa)}$, when we construct $g^{+}$we can arrange that $g^{+} \ni p$. We claim that this suffices to guarantee that $j^{*} g \subseteq g^{+}$. This follows from the observation that $p \leq j(q)$ for every $q \in g$, which is true because $q$ has size less than $\kappa$ and so $j(q)$ is just a copy of $q$.

We can now build $j: V[G][g] \rightarrow M\left[G^{+}\right]\left[g^{+}\right]$, using Lemma 10. Before we can finish the construction, we need one piece of information about this embedding. We claim that

$$
M\left[G^{+}\right]\left[g^{+}\right]=\{j(F)(\kappa) \mid F \in V[G][g] \text { and } \operatorname{dom}(F)=\kappa\} .
$$

To see this let $\dot{\tau}^{G^{+} * g^{+}}$be some element of $M\left[G^{+}\right]\left[g^{+}\right]$, where $\dot{\tau}$ is a $P_{j(\kappa)+1^{-}}$ name in $M$. We know that $\dot{\tau}=j(f)(\kappa)$ for some $f \in V$, and we may as well assume that $f(\alpha)$ is a $\mathbb{P}_{\kappa+1}$-name for every $\alpha<\kappa$. Let us define $F \in V[G]$ by $F(\alpha):=f(\alpha)^{G * g}$. Since $j(G * g)=G^{+} * g^{+}$, we see that $j(F)(\kappa)=(j(f)(\kappa))^{G^{+} * g^{+}}=\dot{\tau}^{G^{+} * g^{+}}$as required.

We will now define a filter $h^{+}$on $\mathbb{Q}_{j\left(\kappa^{+}\right)}$by setting

$$
h^{+}:=\{q \mid(\exists p \in h) j(p) \leq q\} .
$$

It is easy to see that $h^{+}$is in fact a filter, and certainly $j^{\text {" }} h \subseteq h^{+}$and $h^{+} \in V[G][g][h]$. We claim that $h^{+}$is generic. To see this, let $D \in M\left[G^{+}\right]\left[g^{+}\right]$ 
be a dense subset of $\mathbb{Q}_{j\left(\kappa^{+}\right)}$. We know $D=j(F)(\kappa)$ for some $F \in V[G][g]$. Define $E \subseteq \mathbb{Q}_{\kappa^{+}}$by

$$
E=\bigcap\left\{F(\alpha) \mid F(\alpha) \text { is a dense subset of } \mathbb{Q}_{\kappa^{+}}\right\} .
$$

$\mathbb{Q}_{\kappa^{+}}$is $<\kappa^{+}$-distributive, so that $E$ is dense, and clearly $E \in V[G][g]$. Therefore there is some $p \in E \cap h$. Certainly $j(p) \in h^{+}$, and by elementarity $D=j(F)(\kappa) \supseteq j(E)$ so that $j(p) \in h^{+} \cap D$.

In conclusion, we can define $j: V[G][g][h] \rightarrow M\left[G^{+}\right]\left[g^{+}\right]\left[h^{+}\right]$in the model $V[G][g][h]$, so that $\kappa$ is still measurable in $V[G][g][h]$.

5. The collapse. To save on notation, we will now denote the model $V^{\mathbb{P}_{\theta}}$ constructed in Section 3 by $V$. In this model we have the following situation. For all $i$,

(1) GCH holds.

(2) $\lambda_{i}=\operatorname{cf}\left(\lambda_{i}\right)<\lambda_{i}^{+}<\theta_{i}=\operatorname{cf}\left(\theta_{i}\right)<\kappa_{i}$, and $\kappa_{i}$ is measurable.

(3) $\lambda_{i}>\left(\sup \left\{\kappa_{j} \mid j<i\right\}\right)^{++}$.

(4) $\operatorname{SNR}\left(\theta, \lambda_{i}\right)$ holds for every $i$.

We still have to get the reflection property $\operatorname{Ref}\left(\kappa_{i}, \theta_{i},<\theta_{i}\right)$ for every $i$. We will do this by collapsing the measurable cardinals $\kappa_{i}$, using an idea from Section 7 of [2]. We will also check that this collapse does not destroy the strong non-reflection.

Let $\mathbb{S}_{i}:=\operatorname{Coll}\left(\theta_{i},<\kappa_{i}\right)$. We define $\mathbb{S}$ to be the Easton product of the $\mathbb{S}_{i}$; to be precise, $p \in \mathbb{S}$ iff $p$ is a function with

(1) $\operatorname{dom}(p) \subseteq \theta$.

(2) $p(i) \in \mathbb{S}_{i}$ for all $i \in \operatorname{dom}(p)$.

(3) If $\sigma \leq \theta$ is an inaccessible cardinal and $i<\sigma \Rightarrow \kappa_{i}<\sigma$, then $\operatorname{dom}(p) \cap \sigma$ is bounded in $\sigma$.

The ordering is the natural one.

For each $i$, the forcing $\mathbb{S}$ factorises as $\mathbb{S}_{i}^{1} \times \mathbb{S}_{i} \times \mathbb{S}_{i}^{u}$, where $\mathbb{S}_{i}^{1}$ talks about the coordinates below $i$ and $\mathbb{S}_{i}^{u}$ talks about those above. Using Easton's Lemma and the GCH, it is easy to see that $\mathbb{S}$ collapses cardinals in the interval $\left[\theta_{i}, \kappa_{i}\right)$ to $\theta_{i}$ and preserves all other cardinals. In particular, $\operatorname{SNR}\left(\theta, \lambda_{i}\right)$ still holds in $V^{\mathbb{S}}$, because $\lambda_{i}$ is still regular and there are no new points of cofinality $\lambda_{i}$ (this is easy, because by our assumptions on $\lambda_{i}$ we have $\left|\mathbb{S}_{i}^{1}\right|<\lambda_{i}$, and $\mathbb{S}_{i} \times \mathbb{S}_{i}^{u}$ is $\lambda_{i}^{+}$-closed).

For the reflection, it will suffice to check that $\operatorname{Ref}\left(\kappa_{i}, \theta_{i},<\theta_{i}\right)$ holds in $V^{\mathbb{S}_{i}^{1} \times \mathbb{S}_{i}}$, because this model agrees with $V^{\mathbb{S}}$ up to $\theta_{i+1}$. We will look at $V^{\mathbb{S}_{i}^{1} \times \mathbb{S}_{i}}$ in a slightly different way, by writing it as $\left(V^{\mathbb{S}_{i}}\right)^{\mathbb{S}_{i}^{1}}$.

Since GCH holds in $V$ and $\kappa_{i}$ is measurable there, the results of [2] show that $\operatorname{Ref}\left(\kappa_{i}, \theta_{i},<\theta_{i}\right)$ holds in $V^{\mathbb{S}_{i}}$. Of course, $\kappa_{i}$ is now $\theta_{i}^{+}$. We claim that 
$\operatorname{Ref}\left(\kappa_{i}, \theta_{i},<\theta_{i}\right)$ still holds in $\left(V^{\mathbb{S}_{i}}\right)^{\mathbb{S}_{i}^{1}}$. Observe that $\left|\mathbb{S}_{i}^{1}\right|<\theta_{i}<\kappa_{i}$, so that if $S$ is a stationary subset of $S_{<\theta_{i}}^{\kappa_{i}}$ in $\left(V^{\mathbb{S}_{i}}\right)^{\mathbb{S}_{i}^{1}}$ then there is $T \subseteq S$ stationary with $T \in V^{\mathbb{S}_{i}}$. By the reflection which holds in $V^{\mathbb{S}_{i}}$, there is $\gamma \in S_{\theta_{i}}^{\kappa_{i}}$ such that $T \cap \gamma$ is stationary in $V^{\mathbb{S}_{i}}$. Since $\left|\mathbb{S}_{i}^{1}\right|<\theta_{i}$, we see that $T \cap \gamma$ is still stationary in $\left(V^{\mathbb{S}_{i}}\right)^{\mathbb{S}_{i}^{1}}$ (and of course $\gamma$ still has cofinality $\theta_{i}$ ).

We have shown that $\operatorname{SNR}\left(\theta, \lambda_{i}\right)$ and $\operatorname{Ref}\left(\kappa_{i}, \theta_{i},<\theta_{i}\right)$ hold in $V^{\mathbb{S}}$ for all $i<\theta$. This concludes the proof of Theorem 1 .

\section{References}

[1] J. Baumgartner, Iterated forcing, in: Surveys in Set Theory, A. Mathias (ed.), London Math. Soc. Lecture Note Ser. 87, Cambridge Univ. Press, 1983, 1-59.

[2] -, A new class of order types, Ann. Math. Logic 9 (1976), 187-222.

[3] J. Cummings and S. Shelah, Some independence results on reflection (CuSh F129), in preparation.

[4] M. Džamonja and S. Shelah, Saturated filters at successors of singulars, weak reflection and yet another weak club principle, submitted.

[5] A. Kanamori, W. Reinhardt and R. Solovay, Strong axioms of infinity and elementary embeddings, Ann. Math. Logic 13 (1978), 73-116.

INSTITUTE OF MATHEMATICS

THE HEBREW UNIVERSITY OF JERUSALEM

91904 JERUSALEM, ISRAEL

E-mail: CUMMINGS@MATH.HUJI.AC.IL DZAMONJA@MATH.HUJI.AC.IL SHELAH@MATH.HUJI.AC.IL

Current address of Mirna Džamonja:

MATHEMATICS DEPARTMENT

UNIVERSITY OF WISCONSIN-MADISON

MADISON, WISCONSIN 53709

U.S.A.
DEPARTMENT OF MATHEMATICS RUTGERS UNIVERSITY NEW BRUNSWICK, NEW JERSEY 08854 U.S.A. 